\title{
MOLA HIDATIFORME
}

\section{Revisión de 91 casos clínicos en el Hospital Universitario Unidad Obstétrica}

\section{Doctores Fernando Valencia Hurtado ", Humberto Góngora Sánchez "Octavio Vélez Ramírez ${ }^{*: *}$, Fabio Mejía Vieira **, Eduardo Mejía Rojas ${ }^{* * *}$}

Revisión de casos clínicos

El propósito de este trabajo sobre mola hidatiforme es presentar una revisión discriminativa de 91 casos que se han presentado en la Unidad Obstétrica del Departamento de Obstetricia y Ginecología del Hospital Universitario de Caldas, Facultad de Medicina, en el período comprendido entre el $1^{\text {9 }}$ de enero de 1957 al 30 de junio de 1963.

La razón de este estudio es la alta incidencia que entre nosotros supera a casi todas las estadísticas conocidas.

\section{Incidencia}

Se resume en el cuadro siguiente:

CUADRO 1

\begin{tabular}{|c|c|c|c|c|c|}
\hline Año & & Embarazos & Abortos & Molas & Coriocarcinomas \\
\hline 1957 & & 1.728 & 625 & 16 & 1 \\
\hline 1958 & & 1.743 & 430 & 13 & 1 \\
\hline 1959 & & 1.784 & 449 & 16 & 1 \\
\hline 1960 & & 2.404 & 533 & 13 & 0 \\
\hline 1961 & & 2.648 & 542 & 9 & 1 \\
\hline 1962 & & 2.374 & 652 & 20 & 1 \\
\hline \multirow[t]{2}{*}{1963} & (1er. semestre) & ) 1.482 & 289 & 3 & 0 \\
\hline & Totales: & 14.163 & 3.520 & 91 & 5 \\
\hline
\end{tabular}

*Ex-Jefe del Departamento, ** Jefe de la Unidad Obstétrica, *** Profesores Auxiliares de Obstetricia. 
a) Del total de 91 molas, 4 sufrieron degeneración a coriocarcinoma comprobado por Anatomía Patológica.

b) La paciente con coriocarcinoma que figura aparte (1957) ingresó en avanzado estado de evolución con metástasis a pulmones, vagina, etc., y falleció sin que se hubiera comprobado ningún antecedente de mola hidatiforme.

\section{Incidencia comparativa}

No conocemos las estadísticas de ningún hospital del país. Incidencia comparativa con autores que se han ocupado de este estudio: La doctora Bárbara Logan y otros (9) en estudio clínico patológico de mola hidatiforme publica los siguientes datos:

\section{CUADRO 2}

\section{En relación a embarazos:}

Woman's Hospital

Margaret Hague Maternity

New York Hospital

Hertingand Edmonds

Novak

Filipinas, Malaya (según Acosta Sison)

Hospital Universitario. Manizales

$\begin{array}{ll}70.171 & 1 \times 974 \\ 75.000 & 1 \times 1.321 \\ 30.000 & 1 \times 1.400 \\ - & 1 \times 2.062 \\ \square & 1 \times 2.050 \\ 14.163 & 1 \times 145 \\ & 1 \times 155 \quad(0.64 \%)\end{array}$

\section{En relación a abortos:}

Woman's Hospital

New York Hospital

Belleveu Hospital

Mall Collection

Hospital Universitario. Manizales

$\begin{array}{lllll}7.121 & 1 \times 99 & \\ 2.000 & 1 \times 95 & \\ 4.500 & 1 \times 214 & \\ 2.859 & 1 \times & \times 261 & \\ 3.520 & 1 & \times 38 & (.5 \%)\end{array}$

CUADRO 3

Nọ de casos

$\%$

\section{FDAD}

De menos de 20 años

10

De 20 a 29 años

38

De 30 a 39 años

35

De 40 años y más

Totales:

8

11

41.8

38.4

8.8

100.0 
Los casos extremos dan 2 de 18 años y 1 de 49 años, y es natural observar que la mayor incidencia está en el período de máxima actividad genital, o sea en tercer decenio, siguiendo luego el cuarto decenio, después el segundo y por último el quinto.

Lugar de nacimiento

\section{CUADRO 4}

\section{Manizales}

Poblaciones de Caldas

Otros Departamentos

Totai:

\section{Procedencia}

\section{CUADRO 5}

Manizales

77

Poblaciones de Caldas

Totales:

$91 \quad 100.0 \%$
91
Embarazo que comprende la mola

CUADRO 7
Primer embarazo 9

Segundo embarazo 9

Tercer embarazo 6

Cuarto embarazo 12

Quinto embarazo $\quad 7$

Sexto embarazo 5

Séptimo embarazo 8

Octavo embarazo 8

Noveno embarazo 1

Décimo y más embarazos 26

\section{Recidiva}

En nuestro estudio pudimos comprobar la existencia de mola recidivante por haber tenido aborto en condiciones similares. Los reportamos como datos por no tener la evidencia plena.

\section{Sintomatología}

\section{Paridad}

Se resume el cuadro siguiente:

$$
\text { CUADRO } 6
$$

$\begin{array}{lrrr}\text { Primigestantes } & 9 & 9.9 \% \\ \text { Mutigestantes } & 82 & 90.1 \% \\ \text { Totales: } & 91 & & 100.0 \%\end{array}$

Siendo los síntomas principales la metrorragia irregular y prolongada, el flujo achocolatado, la toxemia, hiperemesis, el dolor pélvico, el desarrollo exagerado del útero, la expulsión de vesículas y aun la ausencia de los mismos (ya que sólo hace el diagnóstico la anatomía patológica en las molas asintomáticas), nos permitimos agrupar en cuadro explicativo el porcentaje que hace referencia: 


\section{Síntomas}

Sin metrorragia

a) Metrorragia

b) Flujo achocolatado

c) Toxemia

d) Dolor pélvico

e) Expulsión espontánea de vesículas

f) Asintomáticas (ectópicos)

g) Quistes luteínicos reportados

Siendo de observar que hay casos que presentan más de un síntoma, y por ello figuran en 2 o más veces.

a) Se sabe que una metrorragia, después de corto período de amenorrea en mujer de vida genital activa, es presuntiva de amenaza de aborto. Este aborto será molar cuando las vellosidades tienen degeneración hidrópica macro o microspópica. La metrorragia es a veces insidiosa, larga, y a veces cataclísmica. O sea que pueden reportarse dos períodos: el de amenaza de aborto y el de aborto mismo, donde el dolor pasa a ser un síntoma más llamativo.

b) El flujo achocolatado es signo de poco valor, de bajo porcentaje, que sólo se referirá a perturbaciones cervicales o deciduales.

c) La toxemia gravídica puede presentarse con mayor frecuencia, y es índice de toxicidad de la gonadotropina coriónica, que puede llevar la paciente hasta hacer el cuadro clínico completo, de preferencia después del cuarto mes. Dicho cuadro será agudo, pero no largo y caracterizado por los fenó m e n os de pre-eclampsia; la proteinuria no dará lugar a hipoproteinemia, por ser de duración corta. No habrá secuelas oculares ni marcada hipertensión arterial salvo en las hipertensas y nefríticas pre-gestación.

d) El síntoma dolor merece especial estudio. La gestante en general es aprensiva. Toda mola trae consigo una metrorragia, que se interpreta como aborto, con naturales contracciones uterinas en "terreno" hiperestético. Luego puede aceptarse que haya dolores "vagos" de tipo cólico o sensación de pesantez hipogástrica.

e) La expulsión de vesículas, en el momento del aborto, es el signo que a veces sorprende a quien admite en el hospital la abortante. 
Es el signo patognomónico sobre el cual nada hay que hablar, pues se supone de todos conocido. No obstante, merece consignarse el siguiente concepto: hay quienes refieren haber expulsado "perlas" o "racimos de uvas blancas", o "cirro", o "granizo", expresiones que en el pueblo dicen todo para el médico que atiende consulta de aborto, y con lo cual se puede hacer el diagnóstico retrospectivo o presente.

f) Los llamados casos asintomáticos se refieren a aquellos en los cuales la evolución es tan corta que no da margen a la aparición de las manifestaciones propias. Son quizás los huevos abortivos de Hertig, que en virtud de la degeneración precoz de las vellosidades no alcanzan a hacer implantación, o propiamente fijación en el endo-miometrio, y por ello están dichos huevos abocados a su expulsión rápida, máxime en múltíparas que tengan "cuello incompetente".

La Anatomía Patológica sólo da razón de este diagnóstico, y por este motivo sistemáticamente se envían allí todos los productos obtenidos de legrados uterinos. Esta es la razón del aumento considerable de diagnósticos de mola en nuestro servicio.

g) La coexistencia de quistes llamados luteínicos, con la mola, solo se comprobó en seis casos. De éstos, algunos se dejaron en observación, y la regresión espontánea ocurrió según es habitual en estos casos.

\section{OTROS SIGNOS}

Tamaño del útero como signo:

Ha sido por mucho tiempo y consignado por numerosos autores que la desproporción del desarrollo uterino con el tiempo de embarazo tiene gran valor para diagnóstico positivo de mola. Pues bien, descartando el embarazo múltiple y las afecciones del amnios, aun puede aceptarse que aquél tenga valor, pero no es constante.

Nuestra estadística arroja los siguientes datos:

\section{CUADRO 9}

\begin{tabular}{|c|c|c|}
\hline Tamaño del útero & $N^{o}$ de casos & $\%$ \\
\hline Mayor que la edad & 37 & $40.4 \%$ \\
\hline Igual & 22 & $40.4 \%$ \\
\hline Menor & 10 & $11.2 \%$ \\
\hline Sin informe & 22 & $24.2 \%$ \\
\hline Totales: & 91 & $100.0 \%$ \\
\hline
\end{tabular}




\section{Diagnóstico}

El diagnóstico de mola puede ser presuntivo o definitivo, y éste será macro o microscópico.

El primero se basa en la historia, sintomatología y hallazgos clínicos, asociados a las dosificaciones de la gonadotropina coriónica. El segundo sólo podrá hacerlo con certeza el patólogo.

En Latinoamérica se ha generalizado la prueba cuantitativa de Galli-Mainini como poderoso auxiliar de diagnóstico de mola, y aun se le ha atribuído cierto valor pronóstico, en cuanto a la tendencia degenerativa de la misma, valorando así la actividad corial. Se ha pretendido fijar en ciertos límites lo relativo a embarazo normal (hasta 80.000 unidades) lo pertinente a mola, hasta 200.000 , y cifras superiores a éstas serían índice de carcinoma corial.

La curva de eliminación debe llevarse en forma sistemática a cada paciente (mientras ellas presten dicha colaboración), para poder así determinar si en un momento dado renace la actividad corial, que sí es de valor pronóstico desfavorable fuera de nuevo embarazo.

En nuestro estudio los métodos de diagnóstico utilizados fueron:

$$
\text { CUADRO } 10
$$

\section{Diagnóstico}

\begin{tabular}{lll}
\hline Clínico & 66 & $72.5 \%$ \\
Histopatológico & 72 & $79.0 \%$ \\
Reacciones biológicas & 20 & $22.0 \%$ \\
\hline
\end{tabular}

\section{Localización}

El $97.8 \%$ de los casos fueron de localización uterina. Un $2.2 \%$, de localización tubárica, diagnóstico que fue hallazgo de Anatomía Patológica.

CUADRO 11

\section{Localización}

\begin{tabular}{lrr}
\hline Utero & 89 & $97.8 \%$ \\
Trompas & 2 & $2.2 \%$ \\
\hline \multicolumn{1}{r}{ Totales: } & 91 & $100.0 \%$ \\
\hline
\end{tabular}

Evolución. Pronóstico

La mola requiere expulsarse en aborto molar espontáneo, o mediante la evacuación uterina. En ambos casos puede haber eliminación completa o incompleta; siendo esta modalidad más común, y dando lugar a la persistencia de porciones retenidas que pueden originar sepsis, metrorragias y aun posible degeneración coriocarcinomatosa local o metastásica. La persistencia de estos restos depende de la fijación o invasión que hagan las vellosidades en la decidua, aun en las capas subyacentes del miometrio, constituyendo el "corioadenoma destruens" o mola invasora benigna. Esta condición se traduce en la positividad de la gonadotropina coriónica, cuya curva de eliminación debe llevarse sistemáticamente para un completo control.

El tiempo transcurrido hasta la negativización de las reacciones, en los casos que estamos considerando, fue el siguiente: 
CUADRO 12

Intervalo entre la evacuación molar y la primera prueba biológica negativa

\begin{tabular}{|c|c|c|}
\hline No & de casos & $\%$ \\
\hline Menos de 1 mes & 32 & $35.4 \%$ \\
\hline De 1 a 2 meses & 10 & $10.9 \%$ \\
\hline De 2 a 3 meses & 3 & $3.5 \%$ \\
\hline De 3 a 4 meses & 1 & $1.1 \%$ \\
\hline De más de 4 meses & 1 & $1.1 \%$ \\
\hline Desconocido & 44 & $48.0 \%$ \\
\hline Totales: & 91 & $100.0 \%$ \\
\hline
\end{tabular}

Este elevado porcentaje sin control es falla a nuestro sistema, pues consideramos que con mejor servicio de medicina social (enfermera visitadora, etc.) se mejoraría notablemente el control posterior de estas pacientes.

No debe circunscribirse la evolución a la sola valoración de gonadotropina. Hay otras manifestaciones más objetivas que merecen cuidados especiales, y son las complicaciones inmediatas del aborto molar, como la anemia aguda reportada en alto porcentaje de los casos; toxemia, hipoproteinemia, endometritis aguda y subaguda y aun endometritis sincicial (que fácilmente se confunde con el coriadenoma Destrues), deciduitis y coriocarcinoma.

De estas complicaciones vale la pena detenerse en el análisis de una que puede considerarse intermedia entre mola benigna y mola maligna, y que lleva un sello específico: el infeccioso. Es la endometritis sincicial, o más científicamente la deciduitis sincicial, que asocia el proceso infiltrativo de polimorfonucleares con una cierta capacidad invasora del trofoblasto hacia la profundidad del miometrio. En estos casos se piensa en problema sepsis, por acompañarse de eliminación de flujo purulento a través del canal cervical, conservar una eritrosedimentación alta y leucograma paralelos. Aunque la endometritis sincicial ha sido considerada como entidad benigna, dos de nuestros casos desgraciadamente perdidos de control sufrieron degeneración coriocarcinomatosa, con metastásis generalizadas que hicieron inútil cualquier tipo de tratamiento.

Con el tiempo, si no se tratan activamente, darán lugar a un proceso degenerativo miometrial que dobla el cuerpo uterino en volumen, lo hace friable, sangrante, con invasión parametrial y anexial.

Es $\tan$ frecuente, que la hemos visto reportada en un año cuatro veces en siete molas.

No obstante estas complicaciones presentes en algunos de los casos atendidos, la gran mayoría evolucionó favorablemente: el $50 \%$ de ellas curaron sin secuelas. En 22 de estas pacientes se ha comprobado embarazo posterior.

Se presentaron cinco coriocarcinomas. Tres de estas pacientes fallecieron con invasión a pulmones, hígado, riñones, vulva, etc. En una de ellas no se pudo comprobar antecedentes de mola. En dos por acción diagnóstica, 
combinada con legrados en serie y dosificaciones hormonales específicas, se hizo diagnóstico de degeneración carcinomatosa, por lo cual se realizó histerectomía. Estas pacientes se encuentran actualmente bajo control y en buenas condiciones.

\section{Anatomía patológica}

Desde Hertig se acepta la clasificación histológica de las molas en tres tipos:

I. Aparentemente benigna.

II. Potencialmente maligna, y

III. Aparentemente maligna.

El primer grupo reúne la mayoría de los casos, o sea aquellos en que hay ligera hiperplasia del trofoblasto, edema y escasez de vasos.

En el segundo grupo se consideran aquellas molas en las cuales hay moderada hiperplasia y ligera anaplasia de los elementos trofoblásticos, cierta limitación en el proceso invasor y poca frecuencia metastásica.

En el grupo de las aparentemente malignas, ya existe una marcada anaplasia acompañando a la hiperplasia del trofoblasto, con persistencia de vellosidades más o menos definidas que le imprimen el carácter molar, más la facilidad o frecuencia de metástasis.

La desaparición de las estructuras de las vellosidades inicia el paso de mola a coriocarcinoma.

Por otra parte, se acepta que las vellosidades mismas tienen desigual actividad según el sitio en que se encuentran, siendo mayor en aquellas que van hacia la caduca serotina.

\section{Diagnóstico anatómico}

CUADRO 13

\begin{tabular}{lrr}
\hline Mola benigna & 84 & $92.4 \%$ \\
Mola maligna & 2 & $2.2 \%$ \\
Coriocarcinoma & 5 & $5.4 \%$ \\
\hline \multicolumn{1}{c}{ Totales: } & 91 & $100.0 \%$ \\
\hline
\end{tabular}

CONDUCTA EN CASOS DE MOLA HIDATIFORME

Se refiere a la conducta inmediata de evacuación del tejido molar y al control posterior, para disminuír al máximo los peligros de la mola invasora y del coriocarcinoma.

\section{Tratamiento inmediato:}

Se acepta unánimemente como conducta de elección la pronta evacuación uterina previa dilatación del cérvix, haciendo una revisión digital de Ia cavidad y legrado con cureta roma, enviando a continuación el producto obtenido a estudio anatomopatológico. No sobra recordar el extremo cuidado que se debe tener en estos casos, no sólo en la ejecución del legrado mismo sino también en cuanto se refiere al estado general de la paciente (plasma, sangre, etc.). Si la fibra uterina responde al estímulo, es aconsejable el uso de oxitócicos, que hacen de la evacuación una maniobra más sencilla y menos peligrosa.

Complementos del tratamiento quirúrgico son la radiografía pulmonar y una o dos reacciones cuantitativas de 
Galli-Mainini en el post-operatorio inmediato. Además, se debe practicar el examen pélvico para determinar el grado de involución uterina y la presencia de quistes ováricos.

\section{Control posterior:}

Se basa en el reconocimiento periódico de la paciente y en el estudio de los títulos de gonadotropina en el suero, mediante la reacción de Galli-Mainini cuantitativa.

Estos exámenes se harán tanto más frecuentes cuanto más avance en la clasificación de Hertig. Un intervalo de 15 días para las reacciones se considera prudente al principio, y una vez conseguida la negatividad podrán, según el caso, ser menos frecuentes.

La menstruación puede aparecer en cualquier tiempo, y se diferenciará de una hemorragia patológica por la ausencia de gonadotropina.

Si pasados 30 días de la evacuación molar el título de gonadotropina permanece todavía elevado y acompañado de hemorragia y subinvolución uterina, se aconseja un segundo legrado y unfradiografía pulmonar para referencis futura. Después de este segundo legrado, cuyo producto debe ser analizado en Anatomía Patológica, se continúa estudiando los títulos de gonadotropina, y si aún permanecen altos, se justifica practicar la histerectomía, aun en ausencia de otros síntomas clínicos. Puede tratarse de un corioepitelioma o de un corioadenoma destruens, que por invadir el miometrio queda fuera del alcance de la cureta. El estudio de las gonadotropinas debe continuar después de la histerectomía, hasta obtener el título negativo y en el transcurso de un año.

En el cuadro siguiente se resume el tratamiento dado en los 91 casos considerados:

CUADRO 14

\begin{tabular}{lrr}
\hline $\begin{array}{l}\text { Método de } \\
\text { tratamiento }\end{array}$ & No de casos & $\%$ \\
\hline Legrado simple & 65 & $71.5 \%$ \\
Legrado de repetición & 16 & $17.5 \%$ \\
Histerotomía & 2 & $2.2 \%$ \\
Histerectomía & 6 & $6.6 \%$ \\
Salpingectomía & 2 & $2.2 \%$ \\
\hline \multicolumn{1}{c}{ Totales: } & 91 & $100.0 \%$ \\
\hline
\end{tabular}

\section{CONCLUSIONES}

I. Se hace un estudio discriminativo de 91 casos de mola hidatiforme que se presentaron al Hospital Universitario de Caldas, Departamento de Obstetricia y Ginecología. Unidad Obstétrica. Facultad de Medicina, en el período comprendido entre el $1^{\text {o }}$ de enero de 1957 y el 30 de junio de 1963.

II. Se hace un estudio comparativo respecto a la incidencia de mola en relación con embarazos y con abortos en nuestro hospital y en otros hospitales, haciendo resaltar la elevada incidencia entre nosotros. 
III. Se estudia la mola, desde el punto de vista de edad, paridad y sintomatología, resaltando que el tamaño del útero como signo apenas tiene valor relativo.

IV. En evolución, se insiste sobre la frecuencia de sepsis y de la degeneración maligna.

V. Se sugiere adoptar una clasificación anatomopatológica adecuada de las molas y realizar un estudio completo en todo aborto para descubrir molas microscópicas y coriocarcinomas in situ.

VI. Se insiste en que la reacción de Galli tiene valor auxiliar en estos casos.

VII. Se fija una conducta a seguir en los casos de mola.

VIII. En el embarazo tubárico también existe la transformación molar en las vellosidades coriales.

\section{BIBLIOGRAFIA}

1. ACOSTA-SISON: Am. J. Obst. \& Gynec. Vol. 53. P. 133. Enero, 1957.

2. ACOSTA-SISON: Am. J. Obst. \& Gynec. Vol. 67. P. 634. Marzo, 1954.

3. ACOSTA-SISON: Am. J. Obst. \& Gynec. Vol. 70. P. 666. Septiembre, 1955.

4. ANGEL M. GILBERTO: Reacción de Galli-Mainini. Revista Colombiana de Obstetricia y Ginecología 8 (3): 158-159. 1957.

5. BROW D. B.: A case of co-existing hydatiform mole and living child. Journal of Obstetrics and Gynecology of the British Empire. 64 (3): 446-447. 1957.

6. COPPLESON M.: Hydatiform mole and ist complications. Journal of Obstetrics and Gynecology of the British Empire. 65 (2): 238-252. 1958.

7. CHARVET M. F.: Deux observations d" "hepatonephrite" liées a la existence d'une mole hydatiforme. Bulletin de la Federation des Sociétés de Ginecologie et Obstetrique 9 (2): 170-175. 1957.

8. JORDAN GERMAN y G. LOPEZ ESCOBAR: Rev. Col. de Obst. y Ginec. 10 (1): 35. 1959.

9. LOGAN, B. J. \& MOTYLOFF L.: Hydatidiform mole; a clinical and thological study of 72 cases, with reference to their malignant tendencies. American Journal of Obstetrics and Gynecology. 75 (5): 1134-1148. 1958.

10. NOVAK E. y SEAH C. S.: Am. J. Obst. \& Gynec. Vol. 68. P. 376. Julio, 1954.

11. NOVAK E. y SEAH C. S.: Am J. Obst. \& Gynec. Vol. 67. P. 933. Mayo, 1954.

12. PigEAUD, M. M. \& GABRIEL H.: Nephropathie gravidique precoce. Mole hydatiforme. Bulletin de la Federación des Sociétés de Gynecologie et Obstetrique 9 (5): 581-582. 1957.

13. SCOTT J. S.: Pregnacy toxaemia associated with hidrops foetalis; hydatiforme mole and hydramnios. Journal of Obstetrics and Gynecology of the British Empire. 65 (5): 689-701. 1958.

14. SCHELPERT J. W. \& CONN H.: Hydatiform mole; a survey of 66 cases at Harfod Hospital. American Journal of Obstetrics and Gynecology. 76 (4): 883-893. 1958. 